\title{
CHAPITRE VI
}

\section{LA CONSTRUCTION DES DIALOGUES}

Les travaux des pragmaticiens ont montré qu'une conversation réelle est une construction hiérarchique faite d'unités emboîtées les unes dans les autres, de la plus petite, l'acte de langage, à la plus grande, l'interaction verbale, en passant par des unités intermédiaires (le mouvement, l'échange, la séquence). Ces unités obéissent à chaque niveau à des règles de composition. On dira donc d'une conversation qu'elle est une interaction, composée de séquences thématiques ordinaires, encadrées par des séquences phatiques. Chaque séquence comporte un certain nombre d'échanges constitués de mouvements, initiatifs et réactifs selon qu'ils ouvrent ou ferment l'échange. Chaque mouvement est centré sur un acte de langage principal, accompagné éventuellement d'actes subordonnés. Les échanges sont les unités dialogales fondamentales de la conversation, ils unissent les énonciations de chacun des locuteurs en présence. Ils comprennent au moins deux mouvements ${ }^{1}$ monologaux, parfois trois ${ }^{2}$, l'un initiatif, les autres réactifs, chacun assumé par l'un des locuteurs, une question, une réponse et éventuellement un commentaire par exemple. Le mouvement initiatif exerce un contrôle contraignant sur le mouvement réactif, contrôle syntaxique, sémantique et pragmatique. Il lui impose sa forme, son thème, son acte de langage.

Les échanges peuvent se rassembler en une séquence dotée d'une unité thématique et pragmatique (un même but, un même thème). Une conversation " ordinaire " comprend au moins deux séquences d'ouverture et de clôture, que nous

1. Les pragmaticiens lui préferent souvent le terme d'intervention, mais mouvement est plus dynamique, plus propre à rendre la progression d'une interaction grâce à chaque participant.

2. Les linguistes de l'école genevoise estiment qu'un échange peut s'étendre au-delà de deux ou trois mouvements et peut être constitué d'un nombre $n$. de mouvements, tous dépendants d'un même mouvement directeur initiatif jusqu'à ce qu'un accord se produise entre les locuteurs. Nous ne suivrons pas cette position et préférerons parler dans cette situation de cycle négociatif (intégrant un certain nombre d'échanges binaires ou ternaires). 
appellerons phatiques, encadrant au moins une séquence transactionnelle. Les séquences d'ouverture et de clôture sont plus ou moins ritualisées et strictement codifiées. Elles sont souvent passées sous silence dans un dialogue littéraire. Les séquences transactionnelles constituent l'essentiel de la conversation selon les buts que les participants se sont donnés. À l'intérieur d'une interaction, plusieurs séquences peuvent se succéder et présenter différents thèmes, différents modèles de construction, différents enjeux, offrant aux participants différents rôles discursifs (questionneur, répondeur, réfutateur...) et modifiant leur position hiérarchique l'un par rapport à l'autre.

Nous récapitulerons ces diverses unités hiérarchiques à l'aide d'un tableau, emprunté à Catherine Kerbrat-Orecchioni ${ }^{3}$ :

Unités dialogales :

Unités monologales:
- conversation (interaction)

- séquence

- échange

- mouvement (intervention)

- acte de langage

Doit-on faire des aménagements pour adapter cette hiérarchie au dialogue littéraire? Le plus souvent les dialogues romanesques ne sont, comme l'écrit Sylvie Durrer, que des "bribes d'interaction ${ }^{4}$ ", ne présentant qu'une séquence thématique, centrée sur un seul but, voire un seul échange, dans lequel les rôles discursifs se maintiennent, et passant sous silence les séquences phatiques qui l'encadrent. Il est très rare que deux thèmes de conversation, c'est-à-dire deux séquences se succèdent. Cependant, la notion de séquence reste pertinente pour étudier les enchaînements des répliques, en particulier sur les dialogues longs.

\section{Les répliques}

Paradoxalement, cette description des interactions ne comprend pas la notion de réplique, qui semble pourtant être la plus évidente: réplique n'est pas le terme choisi par les linguistes qui lui préfèrent " tour de parole » ou " intervention ", et le réservent à une catégorie particulière de réponse, celles qui commentent l'énonciation du locuteur précédent ${ }^{5}$. Mais ce mot, consacré par la tradition critique

3. KERBRAT-ORECCHIONI C., op. cit., p. 213.

4. DURRER S., op. cit. p. 95.

5. "D’une manière générale, on utilisera "réaction" comme un terme générique, désignant toute forme de comportement, verbal ou non verbal, suscité par un comportement précédent; et parmi 
littéraire, me semble plus approprié à la spécificité d'un dialogue fictif. Alors que le " tour de parole» est une unité foncièrement monologale de la conversation, la réplique 6 est toujours polyphonique: elle intègre en elle à des degrés divers la voix du narrateur et rassemble parfois en une seule émission la voix de plusieurs personnages. Dans l'exemple suivant, le jugement porté sur Aélis est profondément polyphonique et intègre la voix du personnage à celle du narrateur:

[Ysabiax] cele est a sa mere venue,

Se li a noncié la venue

Et la proiere a la pucele

Ki tant par est cortoise et bele,

Ki demandoit laiens l'ostel.

Ce dist la vielle: «Ainc n’oï tel. » (Escoufle, 4975-4980)

Les paroles d'Isabelle sont 'rapportées'en discours narrativisé qui synthétise les mots 'prononcés' pour n'en garder que la valeur d'usage. Qui " dit » alors le vers 4978 qui contraste par sa forme pragmatique (on passe d'une information à un jugement) et temporelle (on passe du passé au présent)? Le lecteur moderne aura tendance à considérer que la fin de la phrase d'Isabelle: " ki tant par est cortoise et bele,/ki demandoit laiens l'ostel. " n'appartient pas aux paroles de la jeune fille mais au discours du narrateur, malgré la continuité syntaxique. Et ce d'autant plus que ce genre de commentaire laudatif est permanent dans la bouche du narrateur ${ }^{7}$.

C'est pour signaler que nous avons affaire à une construction littéraire que je choisis donc ce terme de réplique qui est au tour de parole ce que le dialogue est à la conversation, un artefact littéraire. La réplique, comme le tour de parole, s'intègre à un échange dans lequel chacun des interlocuteurs parle successivement, sans se confondre complètement avec le mouvement. Elle se situe plus précisément à cheval entre le mouvement et l'échange.

Un tour de parole, ou la réplique qui le représente, peut ne comporter qu'un seul mouvement, ces deux notions se confondent alors:

(a) «Bel enfant, fet ele [Nicolette], conissiés vos Aucassin, le fil le conte Garin de Biaucaire?

les réactions, on distinguera les "réponses" au sens strict, qui apportent une information demandée, et les "répliques" qui commentent l'énonciation de $\mathrm{L}_{1}$. Le terme de "réplique" est donc polysémique, puisque, appliqué au dialogue théâtral (et parfois par extension au dialogue ordinaire), il fonctionne comme un équivalent de "tour de parole". ", KERBRAT-ORECCHIONI C., op. cit., t.1, p. 206.

6. Nous utiliserons ce terme quel que soit le mode de discours rapporté utilisé.

7. Voir par exemple v. 2998, 3787, 3975 ... 
(a') - Ö̈l, bien le counisçons nos. » (Aucassin, I 8, 15-16)

Cet échange didactique est ici constitué d'un premier mouvement (a) initiatif formulé par Nicolette, la question qu'elle pose et d'un deuxième (a'), réactif qui est le fait du berger, sa réponse. Chaque réplique comprend un seul mouvement. Inversement une réplique peut être à cheval sur deux échanges:

(a) [« Mesire Palamidés, fait Kahedins, quel part vaurés vous cevauchier?

(a') - Kahedin, fait Palamidés, il m’en couvient aler vers le roiaume de Gorre, car cele part ai je a faire une moie besoigne qui mout me touce au cuer.] (b) [Et vous, que ferés? Remanrés vous en cest païs u vous irés en la Petite Bretaingne?

(b’) - Certes, sire, fait Kahedins, je m'en irai en la petite Bretaingne au plus tost que je onques porai...]" (Tristan 1, 120, 24-30)

Deux échanges se succèdent ici [aa'] et [bb'], la frontière entre les deux se trouve dans la réplique de Palamède qui est composée d'abord d'un mouvement réactif $(\mathbf{a})$, la réponse à la question de son compagnon, et ensuite d'un mouvement initiatif (b), l'interrogation réciproque de Kahedin. On voit ainsi que la notion de mouvement ne recoupe pas celle de tour de parole, ni de réplique. $\mathrm{La}$ distinction entre la réplique et le mouvement permet de percevoir les techniques de construction des dialogues: dans Aucassin et Nicolette, les bergers sont cantonnés à un rôle réactif, alors que dans le Tristan, les deux chevaliers, situés au même niveau hiérarchique, sont alternativement à l'initiative de chaque échange.

Malgré les apparences, la limite d'une réplique n'est pas toujours facile à déterminer pour l'observateur: dans un texte moderne, un retour à la ligne signale le changement d'interlocuteur; dans les manuscrits médiévaux, aucun signe de ponctuation systématique ne traduit typographiquement le changement d'énonciation. Un certain nombre de procédés - verbe de parole en discours attributif, appellatifs ${ }^{8} \ldots-$ permettent de signaler le seuil du changement d'énonciateur. Mais la notion de réplique est comprise différemment selon les auteurs et les éditeurs. Dans le Merlin, par exemple, l'éditeur est contraint par l'introduction d'un deuxième verbe de parole placé devant à scinder le tour de parole en deux répliques:

Si les mena [Merlins] devant Blaises et li dist: «Voiz ci cels que je te dis qui me devoient venir querre por ocirre ", et dist as messaiges: "Je vos pri..."

(Merlin, 22, 3-6)

8. Voir Cerquiglini B., op. cit. p. 31, et M. PERRET, "Ponctuation et discours rapporté ", GALDERISI C. et MAURICE J. (dir.) "Qui tant savoit d'engin et d'art » : Mélanges de philologie médiévale offerts Gabriel Bianciotto, Poitiers, Presses de l'Université, 2006, p. 359-366. 
Ici, en raison du changement de destinataire, une seule émission de voix est partagée en deux répliques, chacune consacrée à un destinataire. La coupure entre les deux est fortement marquée par le discours attributif.

Mais cette scission de la réplique peut aussi marquer les deux mouvements du tour de parole:

Il la salue, et ele lui, et il li demande s'ele a besoig.

"Oil, fait ele, mout dolereus. Et vos, o alez issi ?

- Damoisele, fait il, ge vois en un mien affaire ou ge n'ai pas encor tant esploitié com ge vousisse. Bele douce amie, fait il, savriez me vos dire novelles del chevalier qui fist antrer lo roi en la Dolereuse Garde? » (Lancelot, p. 629)

Dans cet exemple, le tour de parole est scindé par le discours attributif, en deux répliques, chacune consacrée à l'un des mouvements de l'échange. La première est réactive, la seconde initiative d'un nouvel échange. Si, dans le Lancelot, la disposition typographique moderne n'est pas modifiée, la répétition du verbe de parole et de l'appellatif montre que le narrateur a nettement senti ${ }^{9}$ que cette réplique comportait deux mouvements. Cette scission de la réplique peut même bousculer la continuité syntaxique:

«Sire, fait Galehoz, n'an dotez ja, car ge n'an mantiroie, fait Galehoz, por tot lo reiaume de Logres gaignier. Et ge lo vos fiancerai comme leiaus chevaliers. » (Lancelot, p. 834)

La répétition du discours attributif, dont on peut considérer qu'elle divise le tour de parole en deux répliques malgré la logique syntaxique, pourrait s'expliquer simplement par une leçon défectueuse. Mais ici, comme à chaque fois que l'on trouve une telle répétition, elle peut aussi répondre à une logique pragmatique de mise en valeur: Galehaut s'engage à tenir sa parole et le deuxième verbe de parole n'est pas placé par hasard à côté du verbe mentir, il le met en valeur en soulignant l'aspect promissif de la réponse et marque le seuil du performatif. La suite de la phrase prend alors un aspect prophétique puisque c'est justement au royaume de Logres que renoncera Galehaut pour garder ses engagements auprès de Lancelot. D’une manière générale, la prose ne craint pas ce que nous, lecteurs modernes, sentons comme un excès de discours attributif. Il signale à l'intérieur d'un tour de parole les différentes valeurs illocutoires des propos tenus.

Dans d'autres cas, c'est un changement de registre d'énonciation qui va provoquer la scission d'un tour de parole:

9. ou voulu faire sentir... 
Fait ele: "Tant que j'ai pooir,

Alons en aucune cité

Por manoir. Ml't avons esté

En la queste de mon ami. [...]

Espoir il s'en est retornés

Arriere en la terre sa mere.

Aimi! lasse en cele dont j'ere

N'irai [ja]mais en mon eage,

Car la hautece du lignage

Dont je sui i aroit grant honte."

En plorant dist: "A moi que monte

De mon lignage? c'est du mains,

Quant il m'estuet a mes .II. mains

Gaaignier dont je puisse vivre. " (Escoufle, 5420-5437)

Le complément de manière lié au verbe de parole placé devant entraîne la création d'une nouvelle réplique pour un tour de parole qui ne fait que se prolonger. De tels exemples soulignent l'arbitraire de l'application de nos critères modernes d'organisation pour des textes qui ne s'embarrassent guère de ce genre de détails.

Ces limites empêchent le décompte que nous proposons plus bas d'être parfaitement précis. Celui-ci, bien qu'établi avec le plus de rigueur possible, reste soumis aux aléas d'un discernement subjectif permanent.

\section{Le tempo du dialogue}

Les règles d'enchaînement des répliques donnent sa forme au dialogue mais cette forme dépend aussi du tempo du dialogue, propre à chaque auteur et modifiable selon les contraintes dramatiques. Cette approche stylistique va précéder la démarche pragmatique pour montrer la soumission des dialogues aux contraintes narratives et aux modèles littéraires.

Nous appellerons tempo la vitesse du dialogue, celle-ci est produite par la conjonction de deux phénomènes: la taille de chaque réplique et le nombre de répliques dans un dialogue. Un dialogue qui présente une succession de petites répliques aura un tempo plus rapide qu'un autre constitué de seulement quelques répliques assez longues. Cette vitesse est indépendante du nombre d'informations transmises. Ce tempo contribue à donner au récit son aspect enjoué et vivant. Il varie selon les registres des scènes. 


\section{La taille des répliques}

Les réserves faites sur leur délimitation mises à part, la question de la taille moyenne des répliques ${ }^{10}$ reste pertinente pour notre analyse et met en valeur des oppositions entre les différents textes du corpus retenu.

Ce tableau (pages suivantes) est réalisé à partir de toutes les répliques, quel que soit le type de discours. Dans les textes de ce corpus, les répliques font entre 2 et 9 vers ou équivalents, le plus souvent autour de 5 vers. Cependant, l'exemple du Lancelot montre bien que cette taille moyenne, et par conséquent ce tempo moyen, peut varier à l'intérieur d'un même texte: les épisodes de la Guerre de Gaunes comportent des dialogues au tempo plus lent que l'ensemble du texte et que la séquence de la Douloureuse Garde tout particulièrement. Le Tristan en prose mis à part, les textes les plus récents sont aussi les plus rapides: les romans de Gautier d'Arras et de Chrétien de Troyes sont plus lents que ceux de Jean Renart et le Merlin est plus lent que le Lancelot. Éracle est le texte dans lequel les répliques sont en moyenne les plus longues parce que sa dimension hagiographique entraîne un discours argumentatif qui a besoin d'espace pour être cohérent. Les répliques du personnage principal font en moyenne dix vers et ses discours peuvent monter jusqu'à 83 vers successifs. Dans le Tristan en prose, le poète ou ses divers remanieurs manifestent leur plaisir de créer des discours dans des périphrases polies, des justifications rhétoriques qui ralentissent le rythme du dialogue. Le goût de chaque personnage pour les longues tirades relève de la dimension universitaire de sa construction.

La répartition entre répliques longues, très longues, courtes ou très courtes semble cependant plus intéressante que cette moyenne. Globalement les textes en vers présentent un plus grand écart-type de la taille des répliques, ce qui signifie qu'ils permettent une amplitude de répliques plus large. Inversement, les textes en prose ont un rythme plus régulier, les répliques ont globalement la même taille, assez petite. Cette importance quantitative des répliques longues est peut-être, entre autres, le signe d'une plus grande oralité du texte qui se manifeste par un plus grand goût pour l'art oratoire. Quel média, mieux que la voix en effet, peut

10. Pour pouvoir comparer les vers à la prose, en les ramenant tous à la même unité, j'ai calculé pour chaque texte en prose le rapport entre une ligne de texte et un octosyllabe. La mesure eq. vers (équivalent vers) est la transposition des lignes de prose en groupe de huit syllabes. Je n'ai pas compté les monologues, ni les longs récits de Calogrenant dans Yvain ou de Neronneus dans le Tristan 1, pour pouvoir prendre en compte les dialogues secondaires qui y sont intégrés et qui m’ont semblé plus représentatifs. 
La tailles des répliques:

\begin{tabular}{|c|c|c|c|c|c|}
\hline & Yvain & Éracle & Ille & Escoufle & Vergy \\
\hline $\begin{array}{l}\text { Tempo } \\
\text { moyen (nombre } \\
\text { de vers } \\
\text { par réplique) }\end{array}$ & 6,7 & 9 & 7,4 & 4,6 & 5,27 \\
\hline Écart-type & $\begin{array}{l}8,56 \\
\text { (majo- } \\
\text { rité des } \\
\text { répliques } \\
\text { comprises } \\
\text { entre } 2,4 \text { et } \\
11 \text { vers) } \\
\end{array}$ & $\begin{array}{l}13 \\
\text { (majo- } \\
\text { rité des } \\
\text { répliques } \\
\text { comprises } \\
\text { entre } 3,5 \text { et } \\
15 \text { vers) } \\
\end{array}$ & $\begin{array}{l}9,12 \\
\text { (majo- } \\
\text { rité des } \\
\text { répliques } \\
\text { comprises } \\
\text { entre } 2,8 \text { et } \\
12 \text { vers) } \\
\end{array}$ & $\begin{array}{l}6,3 \\
\text { (majorité } \\
\text { des répliques } \\
\text { comprises } \\
\text { entre } 1,5 \text { et } \\
7,8 \text { vers) }\end{array}$ & $\begin{array}{l}5,33 \\
\text { (majo- } \\
\text { rité des } \\
\text { répliques } \\
\text { comprises } \\
\text { entre 2,6 et } \\
5,9 \text { vers) } \\
\end{array}$ \\
\hline $\begin{array}{l}\text { très petites répli- } \\
\text { ques: } \\
\text { ( } \leq 1 \text { vers) }\end{array}$ & $17,3 \%$ & $13,6 \%$ & $18,1 \%$ & $14,3 \%$ & $12,7 \%$ \\
\hline $\begin{array}{l}\text { petites répliques: } \\
\text { (entre } 1,5 \\
\text { et } 5 \text { vers) }\end{array}$ & $39,8 \%$ & $38,7 \%$ & $33,3 \%$ & $57,2 \%$ & $36,4 \%$ \\
\hline $\begin{array}{l}\text { Total des répli- } \\
\text { ques petites } \\
\text { et très petites }\end{array}$ & $57,1 \%$ & $52,3 \%$ & $51,4 \%$ & $71,5 \%$ & $49,1 \%$ \\
\hline $\begin{array}{l}\text { répliques moyen- } \\
\text { nes: (entre } 5,5 \\
\text { et } 9 \text { vers) }\end{array}$ & $19,6 \%$ & $18,3 \%$ & $24,3 \%$ & $16,7 \%$ & $32,7 \%$ \\
\hline $\begin{array}{l}\text { répliques } \\
\text { longues: (entre } \\
9,5 \\
\text { et } 18 \text { vers) }\end{array}$ & $15,3 \%$ & $14,9 \%$ & $16 \%$ & $7,4 \%$ & $12,7 \%$ \\
\hline $\begin{array}{l}\text { répliques très } \\
\text { longues: } \\
\text { ( } \geq 18 \text { vers) }\end{array}$ & $8,2 \%$ & $14,5 \%$ & $8,3 \%$ & $4,4 \%$ & $5,5 \%$ \\
\hline $\begin{array}{l}\text { Total des répli- } \\
\text { ques longues et } \\
\text { très longues }\end{array}$ & $23,5 \%$ & $29,4 \%$ & $24,3 \%$ & $11,8 \%$ & $18,2 \%$ \\
\hline
\end{tabular}




\begin{tabular}{|c|c|c|c|c|c|}
\hline Épée & Aucassin & Pontieu & Merlin & Lancelot & $\begin{array}{c}\text { Tristan } 1 \\
\text { et } 2\end{array}$ \\
\hline 5,8 & 4,9 eq. & 2,1 eq. & 5,3 eq. & $\begin{array}{l}4,7 \text { eq. } \\
\text { épisode de } \\
\text { la guerre de } \\
\text { Gaunes: } 7,88 \\
\text { eq; } \\
\text { épisode de la } \\
\text { Douloureuse } \\
\text { Garde: } 2,75 \mathrm{eq}\end{array}$ & 7,8 eq. \\
\hline $\begin{array}{l}7,46 \\
\text { (majorité } \\
\text { des répliques } \\
\text { comprises } \\
\text { entre } 2 \text { et } 8 \\
\text { vers) }\end{array}$ & $\begin{array}{l}6,24 \\
\text { (majorité } \\
\text { des répliques } \\
\text { comprises } \\
\text { entre } 1,8 \text { et } 8 \\
\text { eq. vers) }\end{array}$ & $\begin{array}{l}1,12 \\
\text { (majo- } \\
\text { rité des } \\
\text { répliques } \\
\text { comprises } \\
\text { entre } 1,5 \text { et } \\
2,7 \text { eq. vers) }\end{array}$ & $\begin{array}{l}4,05 \\
\text { (majorité } \\
\text { des répliques } \\
\text { comprises } \\
\text { entre } 3,3 \text { et } \\
7,3 \text { eq. vers) }\end{array}$ & $\begin{array}{l}4,85 \\
\text { (majorité } \\
\text { des répliques } \\
\text { comprises entre } \\
2,3 \text { et } 7,1 \text { eq. } \\
\text { vers) }\end{array}$ & $\begin{array}{l}4,25 \\
\text { (majorité } \\
\text { des répliques } \\
\text { comprises } \\
\text { entre } 5,7 \text { et } \\
10 \text { eq. vers) }\end{array}$ \\
\hline $11,5 \%$ & $29,3 \%$ & $31,7 \%$ & $14,6 \%$ & $28,3 \%$ & $8,5 \%$ \\
\hline $49,2 \%$ & $36,6 \%$ & $64,1 \%$ & $47,4 \%$ & $45 \%$ & $42,3 \%$ \\
\hline $60,7 \%$ & $65,9 \%$ & $95,8 \%$ & $62 \%$ & $73,3 \%$ & $50,8 \%$ \\
\hline $18 \%$ & $17 \%$ & $2,8 \%$ & $17 \%$ & $14,5 \%$ & $21,4 \%$ \\
\hline $13,1 \%$ & $12,2 \%$ & $1,4 \%$ & $12,4 \%$ & $7,7 \%$ & $20,8 \%$ \\
\hline $8,2 \%$ & $4,9 \%$ & $0 \%$ & $8,6 \%$ & $4,5 \%$ & $7 \%$ \\
\hline $21,3 \%$ & $17,1 \%$ & $1,4 \%$ & $21 \%$ & $12,2 \%$ & $27,8 \%$ \\
\hline
\end{tabular}


prendre en charge les paroles de personnage? La performance orale du jongleur vient ici renforcer celle des personnages. Dans le Lancelot, les répliques longues sont exceptionnelles: malgré la taille impressionnante des discours de la dame du Lac à Lancelot, de Claudas sur le corps de son fils, ou du prud'homme à Arthur, elles ne constituent que 4,5\% de l'ensemble des répliques. Inversement les répliques très courtes sont plus nombreuses dans ce roman puisqu'elles atteignent plus d'un quart des répliques. De même les textes courts sont aussi constitués plutôt de répliques courtes quel que soit le style utilisé. La brièveté du texte s'accompagne d'une condensation des répliques.

La taille des répliques entraîne forcément certaines modifications dans leur écriture même. Les répliques longues sont constituées de mouvements complexes, intégrant plusieurs actes de langage. Alors qu'une réplique courte élimine ou résume sensiblement tout acte de langage secondaire, à valeur de justification ou de commentaire, une réplique longue a tendance à présenter la structure d'un discours, comportant plusieurs points de passage. La comparaison de deux répliques dans un même topos de conversation (dialogue nocturne entre une femme et son mari dans l'Escoufle; son amant dans le Lancelot) permet de montrer d'un côté une construction fortement teintée de rhétorique et de l'autre une condensation du discours sur les seuls actes de langage principaux: 


\begin{tabular}{|c|c|}
\hline Escoufle, répliques longues & Lancelot, répliques courtes \\
\hline $\begin{array}{l}\text { "Sire, fait ele, ja vos ain ge } \\
\text { Plus que mon cors et plus que m'ame. " } \\
\text { Ahi! Ahi! feme que fame } \\
\text { Com le set ore bien atraire! } \\
\text { Je cuit qu'ele li fera faire } \\
\text { Que ses gens faire ne li porent. } \\
\text { "Sire, fait ele, quant il orent } \\
\text { Geü bras a bras longhement, } \\
\text { Car me dites, por Dieu, comment } \\
\text { Est hui primes venue avant } \\
\text { La parole de vostre enfant } \\
\text { Que Guilliaume aime. Je sai bien, } \\
\text { Certes, ml't desirent son bien } \\
\text { Cil qui heent le mariage, } \\
\text { Por ce qu'il n'est pas de lignage } \\
\text { Qu'il la doie penre n'avoir; } \\
\text { Et neporquant, por nul avoir } \\
\text { Ne remandra qu'il ne soit fait } \\
\text { Dés qu'il vos siet et il vos plaist. } \\
\text { Je sui feme qui n'en puis mais, } \\
\text { Si le m'estuet souffrir em pais; } \\
\text { Mais ml't me grieve toute[s] voies. » } \\
\text { Tant li moustre essamples et voies } \\
\text { De raison et tant li cort seure } \\
\text { Et en tel point et en tele eure } \\
\text { Que nus ne li peüst veer } \\
\text { Chose qu'ele vousist rouver. } \\
\text { (Escoufle, 2878-2903) }\end{array}$ & $\begin{array}{l}\text { Et ele s'encomm[anç]a a plaindre a lui de } \\
\text { sa mesaise et dist: } \\
\text { «Sire, vos i deüssiez bien metre consoil, } \\
\text { car ge n'ai toz cels maus se por vos non. } \\
\text { - Certes, fait il g'i metroie mout volen- } \\
\text { tiers consoil se ge savoie. } \\
\text { - Ge vos enseignerai, fait ele, coment vos } \\
\text { me poez de lui vengier et vos avocques, } \\
\text { se vos m'amez tant comme j'ai deservi } \\
\text { envers vos. } \\
\text { - De ce, fait il, seiez vos tote seüre que, se } \\
\text { ge puis avoir lo point, ge vos en vengerai; } \\
\text { et dites comment, se vos saviez, et ge } \\
\text { vos otrois qu'il en iert a vostre voloir. " } \\
\text { (Lancelot, p. 104) }\end{array}$ \\
\hline
\end{tabular}

Dans l'Escoufle, le long tour de parole de l'impératrice est construit essentiellement sur un acte directeur, une requête, formulée en discours narrativisé: «chose qu'ele vousist rouver». Cette requête repose sur un acte secondaire de reproche " $\mathrm{Ne}$ remandra qu'il ne soit fait/Dès qu'il vos siet et il vos plaist. ", et s'accompagne d'actes secondaires à valeur d'arguments, "les essamples et voies de raison" du discours narrativisé, explicités dans le discours direct "ml't desirent son bien,/Cil qui heent le mariage,/Por ce qu'il n'est pas de lignage. " Ces arguments sont pris en charge 
dans une construction rhétorique du discours qui commence par une captatio benevolentic où la dame affirme son amour pour son interlocuteur et se termine sur le topos éthique de la modestie de l'orateur: "Je sui une feme qui n'en puis mais». La réplique longue est donc complexe au point de vue de son organisation illocutoire.

En revanche dans le Lancelot, ces deux actes de langage sont simplement coordonnés: la requête, acte de langage directeur "Sire, vos i deüssiez bien metre consoil ", justifiée par une information "car ge n'ai toz cels maus se por vos non" légèrement accusatrice. De même les répliques qui suivent, qu'elles soient le fait de Claudas ou de la femme de Pharien sont centrées sur un seul acte de langage: Claudas répond tout d'abord par une décision qui marque son accord, il l'assortit d'une demande discrète "se ge savoie " qui oblige sa compagne à s'engager. La dernière réplique de Claudas, qui équivaut à peu près à six vers, contient nettement deux mouvements distincts: tout d'abord la réponse à l'acte d'engagement de la dame, il s'engage en retour " ge vos en vengerai. ", puis une demande d'information. La petite taille d'une réplique entraîne donc un resserrement autour des actes de langage directeurs et le déploiement du propos sur les répliques suivantes tout au long du dialogue.

\section{Les répliques courtes}

Les répliques courtes sont différemment distribuées selon les textes. Dans les textes en vers ou dans Aucassin et Nicolette par exemple, elles ont souvent une fonction de seuil marquant la coupure avec le récit: en début de conversation, elles reçoivent une fonction phatique; en fin de conversation, elles servent à marquer l'acquiescement de l'interlocuteur, généralement celui qui se trouve en position hiérarchiquement basse. Cet acquiescement rapide permet d'achever l'interaction sur un accord:

«Dame, fait il, Dieus soit od vos!

- Vallés, cil Deus qui maint sor nos

Vos salt et gart et beneïe!

- Dame, se Dieus vos face aïe,

Qui est le mescine au bliaut?

- Biaus dous amis, se Dieus me salt

Ce fu la fille au plus rice home

Qui onques esteüst en Rome...» (Éracle, v. 2619-2626)

Uns cevaliers le regarda, si vint a lui, si l'apela:

«Aucassins, fait il, d'ausi fait mal con vos avés ai ja esté malades. Je vos donrai bon consel, se vos me volés croire.

- Sire, fait Aucassins, grans mercis; bon consel aroie je cier. 
- Montés sor un ceval, fait il, s'alés selon cele forest esbanoiier; si verrés ces flors et ces herbes, s'orrés ces oisellons canter; par aventure orrés tel parole dont mix vos iert.

- Sire, fait Aucassins, grans mercis; si ferai jou. "(Aucassin, 20, 19-29)

La première réplique d'Éracle constitue une salutation et permet d'engager la conversation sous de bons augures. La dernière réplique d'Aucassin contient un acte de langage expressif, (un remerciement) et un acte de langage promissif, (la décision du jeune homme). Elle permet de clore le dialogue sur un accord et une prise en compte des propos tenus. En fin de dialogue, la réplique d'un seul vers permet à Gautier d'Arras de refermer son dialogue sur un couplet de vers renforçant ainsi son unité:

Signor et Dix vos beneïe!

Por querre a vos trestos aïe

Me sui penee et travillie,

Qi n'en sui preu consillie

J'ai en Bretagne puis esté,

Mais ke n’i ai rien conquesté

Fors que traval et paine et mal:

Trover cuidé le senescal

Nel trové pas, ce poise moi.

A Saint Jake est alés, je croi,

Issi le dient el païs.

Mon més d'ilueques li tramis,

Mout li rovai qu'il se coitast

Et du revenir se hastast.

Et se li dus cha revenoit

Et vers les Grius nos maintenoit,

Tost en feroie le conseil

De vous qui estes mi feel,

Mi home liege et mi juré

- Damoisele, ça trop duré."

Font se li .x. qui mout sont fier. (Ille, v. 6139-6159)

Intégrée à l'intérieur d'un dialogue, la réplique d'un seul vers, qui implique forcément une brisure du couplet est aussi un bon moyen d'attirer l'attention sur une phrase essentielle. Ceci ajoute un instrument de " mise en relief " à la palette dont disposent déjà les auteurs qui, comme Gautier d'Arras ou Chrétien de Troyes, n'ont pas généralisé la brisure du couplet, ainsi lors des retrouvailles des héros dans Ille et Galeron: 
Biax sire, por Diu, ne t'anuit que t'ont chi avoec moi veü. Li desiriers que j'ai eü d'a toi parler a mon vivant me fist hardie maintenant. Et por Diu, ne m'oblier mie; car je fui ja ta bone amie.

- Comment, ne mamés vos encore?

- Biax sire, se je disoie ore comment je t'aim, quel qui me prenge, on i poroit noter losenge. (ibid., v. 4189-4199)

La réplique où Ille demande à celle qui fut son épouse de lui confirmer son amour est mise en relief par sa brièveté et par la brisure du couplet qu'elle entraîne.

En définitive, y a-t-il une relation "naturelle » entre le vers et la réplique? Peut-on affirmer, comme le fait Christine Ferlampin-Acher, que l'alternance des voix est facilitée par le rythme de l'octosyllabe, "le rythme du vers (plus que sa présentation matérielle) structurait efficacement les échanges ${ }^{11}$ ? » Les auteurs modèlent-ils la réplique sur le vers, ou désynchronisent-ils ces deux rythmes par des changements de tour de parole situés au milieu des vers? Une petite étude statistique montre que dans l'ensemble, les auteurs font coïncider la fin d'une réplique au discours direct avec la fin d'un vers et construisent les répliques sur des vers entiers: le changement d'énonciateur au milieu d'un vers n'atteint que $5 \%$ des répliques chez Gautier d'Arras ; $10 \%$ dans Yvain; $17 \%$ dans l'Escoufle.

Plus souvent (environ $15 \%$ des répliques au discours direct), la réplique ne commence pas tout de suite au début d'un vers, mais un verbe de parole placé devant occupe la première place. La proportion de ce procédé ne varie guère selon les auteurs, mais on peut voir une évolution dans la taille de ce discours attributif. Gautier d'Arras ne lui fait que rarement dépasser les quatre syllabes, il y place peu de noms propres et privilégie l'emploi des pronoms. Le début de la réplique commence par un terme marquant fortement le discours direct: $O r$, mar, ou un appellatif...:

Li dus tramet por la pucele

Et se li dist: «Amie biele... » (Ille, v. 1515-1516)

Lors dient: «Dix, par ton saint non...» (ibid., v. 4998)

11. FERlampin-ACHER Ch., "La Parole dans le Merlin de Robert de Boron », QUÉRUEL D. et FERLAMPIN-ACHER Ch. (dir), Merlin, roman du XIII siècle, Paris, Ellipses, p. 101. 
Tout ce qui vise à développer le discours attributif se trouve dans le vers précédent. En revanche, chez Jean Renart, il s'étend aux dépens des paroles de personnages et occupe la majorité des syllabes du vers. Le discours direct n'est plus une unité infrangible, il est fortement perméable à la voix du narrateur, que celle-ci soit placée avant la réplique ou en incise:

Diex, font cil de l'ost qui le voient,

Quel chevalier et quel preudome! (Escoufle, v. 1020-1021)

Cil est si liés qu'il ne loist

A respondre por sa lïece

plus tost qu'il pot, a dit: "Ou es ce

Que ma damoisele me mande?»(ibid., v. 3344-3347)

Ele a dit ml't cortoisement

As meschines et as puceles

Ki devant li gisent: «Chaieles!

Beles, car levés vos hui mais. " (ibid., v. 3270-3273)

Chez Gautier d'Arras, la coupure est nette: le discours direct est séparé du reste de la narration en suivant l'articulation du vers. Quand le vers commence, on est déjà dans une voix autre, même si elle contient encore quelques (quatre) syllabes de discours attributif placé devant. Malgré son appartenance à la voix du narrateur, son insignifiance sémantique le rend presque transparent: il participe à la même logique que le discours direct, il est de l'autre côté, il n'appartient pratiquement plus à la voix du narrateur. Chez Jean Renart au contraire, la voix du narrateur dispute à celle des personnages la domination du vers, le changement de locuteur n'est plus rythmé par la structure régulière de l'octosyllabe.

Dans la prose, le discours attributif fonctionne comme une véritable bouée empêchant la réplique courte de disparaître. Loin d'empêcher l'évolution des répliques vers l'interdépendance, lui seul permet, dans les conditions de mise en page manuscrite que l'on sait, de progresser vers une plus grande intégration des répliques à un tissu dialogal. Si l'on compare la construction de deux petites répliques dans le long dialogue entre Lancelot et la reine, on voit bien le rôle d'ancrage que fournit le discours attributif en dehors de toute marque énonciative de dialogue:

(1) « Et qant vos fustes partiz de nos a la darriene foiz, o alastes vos?

- Dame, aprés un chevalier que ge sivoie.

- Et combatites i vos?

- Dame, ö̈l.» (Lancelot, p. 884)

(2) « Dites moi, fait ele, et tot seürement que ge ne vos descoverrai ja. Et ge sai bien que por aucune dame ou por aucune damoisele avez vos ce fait. Et dites moi qui ele est, par cele foi que vos me devez. 
- Ha! Dame, fait il, bien voi que il lo me covient a dire. Dame, ce iestes vos.

- Ge? fait ele.

- Voires, dame." (ibid., p. 886)

Dans le premier exemple, l'appellatif sert de seuil et permet, outre son rôle de marqueur de politesse, de désigner le changement d'énonciateur. Dans le second cas, la petite interrogation étonnée de la reine, reprenant en écho cette révélation essentielle, s'appuie sur le discours attributif pour exister. La reine n'emploie en effet que trois appellatifs pour désigner Lancelot tout au long de cette conversation. Ce choix narratif, signifiant au niveau de la construction de la relation interpersonnelle, oblige le narrateur à compenser par une plus forte utilisation du discours attributif.

Dans les textes en vers, la question est compliquée par la prise en compte de la prosodie: les répliques de taille inférieure au vers, quand cette petite taille n'est pas compensée au sein du vers par le discours attributif, entraînent en effet son bouleversement. Une disjonction s'opère entre les différents rythmes du vers, de la syntaxe et de la parole ${ }^{12}$ :

«Dame, fet il, la force vient

De mon cuer, qui a vous se tient;

En cest voloir m'a mon cuer mis.

- Et qui le cuer, biaus dous amis?

- Dame, mi oil. - Et les oilz, qui?

- La grant biautés quë en vous vi.

- Et la biautez, qu'i a forfayt?

- Dame, tant quë amer me fait.

- Amer? Et qui? - Vous, dame chiere.

- Moy? - Voire voir. - En quel maniere?

- En tel que graindre estre ne puet..." (Yvain, v. 2017-2027)

Dans ce passage, les deux répliques qui divisent les vers 2021 et dans une moindre mesure 2025 respectent pour l'essentiel le rythme naturel du vers, le changement d'interlocuteur produisant un balancement binaire qui ne choque pas. En revanche, la réplique monosyllabique du vers 2026 pose un problème de lecture: soit le lecteur respecte le rythme du vers et réunit au moins les deux premières répliques du vers dans une seule émission de voix, au détriment du sens et de l'énonciation; soit il donne la priorité à l'énonciation et place une pause supplémentaire à l'intérieur du vers. Trois pauses, aussi courtes soient-elles, à l'intérieur de l'octosyllabe, commencent à mettre sérieusement en question son

12. Sur ces rythmes, voir BAUMGARTNER E., "Remarques sur la prose du Lancelot ", Romania $n^{\circ} 105,1984$, p. 1-15. 
unité. Ces jeux poétiques remettent en cause l'idée même de vers, à moins qu'on ne considère au contraire qu'ils ne le magnifient. De tels effets ne fonctionnent que si l'idée de vers persiste dans l'horizon d'attente de l'auditeur et si sa forme se maintient à l'oreille, sans quoi aucune tension ne se crée, et nul effet poétique ne se produit. Trois cadres ici se superposent: celui du dialogue qui demande que l'on distingue les voix des locuteurs, celui de la syntaxe et celui de la prosodie.

La tension produite par cette opposition entre trois logiques crée une vibration poétique remarquable mais explique qu'un tel type d'enchaînement ne puisse durer très longtemps à moins d'aboutir à la disparition de l'un de ces trois éléments. Elle renvoie à la tension provoquée par la synthèse de plusieurs modèles de dialogues - le dialogue amoureux qui reprend tous les stéréotypes de la lyrique courtoise et le dialogue judiciaire qui permet le tempo rapide de l'interrogatoire - et contribue comme l'écrit Ph. Ménard à la création d'une sorte de " bulle euphorique ":

Lauteur ne cherche guère à traduire une sorte de harcèlement pressant ou pathétique mais semble se détacher de sa matière pour jongler avec les mots et les répliques. En cultivant le rythme haché, en l'étendant avec complaisance sur plusieurs vers, l'auteur cherche à faire montre de sa virtuosité; l'accélération du mouvement est une rupture délibérée avec le tempo naturel de la vie. Elle nous transporte dans un autre monde, plus léger, plus aérien, où les paroles jaillissent promptement, où les mots se précipitent les uns sur les autres, où par vitesse, l'homme s'affranchit semble-t-il de l'espace et du temps ${ }^{13}$.

Mais, contrairement à $\mathrm{Ph}$. Ménard, qui s'appuie aussi sur des extraits d'Éracle (292-304), je doute que cette vision des choses puisse s'adapter à d'autres exemples que ce poème à deux voix construit par Chrétien de Troyes. Cette interprétation séduisante ne doit pas faire oublier en effet que d'une part, de tels enchaînements de vers courts ou très courts s'étendent rarement sur plus de deux vers, ce qui produit un effet de rupture réel mais trop bref pour créer un rythme: dans Ille et Galeron ${ }^{14}$ on ne trouve que trois fois trois répliques d'un vers qui se succèdent; dans Éracle, il n'y a jamais plus de deux répliques courtes successives. Dans Yvain ${ }^{15}$, qui a pourtant la réputation d'être un roman aux dialogues très vifs, seuls deux enchaînements dépassent les deux répliques courtes. Dans l'Escoufle ${ }^{16}$, on trouve bien deux enchaînements de trois répliques courtes mais un seul qui aille au-delà

13. MÉNARD Ph., Le Rire et le Sourire, p. 742-745.

14. Ille et Galeron: Ille et le Pape, v. 3663-3665 ; un messager de Ganor, les hommes d'Ille, v. $6407-$ 6409; Ille et Ganor, v. 6509-6511.

15. Yvain: Lunette et Laudine, v. 1819-1821; Yvain, Laudine v. 2020-2027.

16. Escoufle: Guillaume et un bourgeois v. 6394-6395; Guillaume et un autre bourgeois v. 65526555: sept répliques en quatre vers; le fauconnier et le comte de Saint-Gilles, v. 7273-7274. 
et qui monte à sept répliques très rapides. D'autre part, ces enchaînements s'inscrivent souvent dans des contextes graves où le ton des dialogues est inquiet voire angoissé ${ }^{17}$ : cette accélération marque la stupeur (dialogue d'Ille avec le Pape, les retrouvailles d'Ille et de Ganor), l'urgence (le messager de Ganor auprès de l'armée d'Ille). Inversement, ce tempo rapide peut être le fait de personnages secondaires et les grands dialogues essentiels à l'intrigue en sont dépourvus. Le cas du dialogue de Guillaume avec le bourgeois qui l'embauche est flagrant:

Fait il: "Comment avés a non?

- Guilliaume. - Dont? - sire, de Toul.

- En es ce en Loherainne? - Aoul.

- Diex! fait li ostes, g’i fui ja. » (Escoufle, v. 6552-6555)

Ce tempo rapide n'a pas de signification psychologique particulière: il permet surtout de styliser le dialogue, de le condenser tout en maintenant le discours direct c'est-à-dire en conservant le pittoresque d'une parole vivante. Ces enchaînements permettent la schématisation du dialogue sans perdre les valeurs dont peut être porteuse la voix des personnages. Même « retranscrits » en discours direct, ces dialogues brefs au tempo très rapide ne sont qu'un raccourci. Le tempo rapide n’a rien à voir ici avec une dimension mimétique ou poétique. Il est le plus souvent un abrégé du « discours réel ».

La situation est un peu différente dans le Lancelot, en particulier dans l'épisode de la Douloureuse Garde ${ }^{18}$ où les tout petits dialogues, qui ne dépassent guère les six répliques et ne s'étendent jamais sur plus de quatre lignes, sont la règle. Beaucoup de ces dialogues concernent des personnages secondaires, gardes, portiers, chevaliers anonymes. Le roi Arthur y est plongé dans une rêverie profonde et Lancelot reste sans voix quand il contemple la reine. Cela ne constitue pas une situation propice au dialogue! Toutefois, cette démultiplication des petits dialogues nous semble surtout due à la technique de composition utilisée dans ces passages : le narrateur se disperse sur plusieurs personnages et essaye de n'en perdre aucun. Le point de vue se déplace sur chacun d'entre eux en une multitude de petites saynètes qui visent à rendre la complexité de la situation: le narrateur est obligé de décomposer au maximum la scène. Ce fractionnement participe de sa volonté, visible par l'entrelacement des scènes, de raconter des situations simultanées. Cet épisode

17. On peut penser aussi au passage de Béroul où un espion vient avertir les trois barons félons de la venue de Tristan dans la chambre d'Yseut (4295-4308). Le tempo rapide de ce passage renvoie plus à la précipitation des participants qu'à un détachement de toutes les règles dramatiques au profit d'une pure poésie.

18. J'étudie ici tout particulièrement les pages 554-588, soit les chapitres XXVII à XXIX. 
qui rassemble les principaux personnages de ce début du roman est une occasion de mener cet entrelacement au maximum de ses possibilités, il ne se fait pas au niveau des chapitres, mais au niveau des lignes de texte, des micro-structures du récit. Le tempo très rapide des dialogues participe à cet entrelacement, les paroles de personnages éclatent en une multitude de micro-événements, caractéristiques de cette " écriture de l'attente » dont a parlé Christine Ferlampin-Acher ${ }^{19}$, c'està-dire la volonté de démultiplier les épreuves et les obstacles imposés. De même que la résolution des mystères de la Douloureuse Garde est retardée par une série d'obstacles, de même la véritable rencontre entre Lancelot et la reine est retardée par des piétinements devant les portes. Cette relation complexe met en jeu tout l'équilibre du royaume arthurien représenté ici par une multitude de personnages emblématiques dont l'auteur ne veut passer sous silence aucun des gestes.

Le tempo rapide ne se limite pas cependant à cet épisode du roman. En effet, il présente de nombreux dialogues très vifs qui s'étendent sur plusieurs répliques et concernent quasiment tous les dialogues secondaires ou essentiels. L'utilisation systématique des incises permet de prolonger ces duels verbaux sans courir le risque d'une déperdition du sens. Rien n'empêche plus que de tels enchaînements se prolongent. C'est le signe que le centre d'intérêt du dialogue s'est déplacé: le ton est autant en jeu que l'exposé des arguments de chaque personnage. Sa forme prend autant d'importance que le fond: la comparaison entre l'aveu d'Ille auprès du Pape et celui de Lancelot face à Guenièvre le montre ${ }^{20}$. Alors qu'llle, certes homme fait et sûr de lui, expose sa situation dans un long discours cohérent, Guenièvre doit arracher un à un les mots de la bouche de son jeune amoureux transi. Passées les trois répliques courtes qui marquent la stupéfaction du pape et qui servent de transition entre l'exposé des réticences d'Ille et celui de son histoire, l'émotion ne modifie pas la forme ni le rythme du dialogue. En revanche, la timidité de Lancelot perturbe fortement sa prise de parole. L'émotion du jeune chevalier a désormais une réelle incidence sur la forme du dialogue. Celle-ci manifeste dans son écriture même ce que le vers se contente d'affirmer.

\section{Les répliques longues}

Les répliques longues ne semblent pas avoir de règle d'emploi particulière, elles intègrent le plus souvent des actes de langage informatifs, expliquant une

19. FERLAMPIN-ACHER Ch., "La Douloureuse Garde du Lancelot en prose: les clefs du désenchantement ", POMEL F., (dir.) Les clefs des textes médiévaux: pouvoir, savoir et interprétation, Rennes, Presses Universitaires de Rennes, 2006, p. 175-192.

20. Pour une étude des modèles différents qui informent ces dialogues, voir infra p. ??? et???. 
situation qui a besoin d'espace pour être formulée, dans un but didactique ou pour justifier une requête ou une décision.

Comme le montrait K. Rogger ${ }^{21}$ sur Aucassin et Nicolette, elles sont souvent construites avec une structure fermée, où une phrase de conclusion vient rappeler l'idée initiale. Lorsque Éracle et sa mère se séparent (Éracle, 571-632), celle-ci commence sa longue plainte (36 vers) en insistant sur la monstruosité de son geste: "C'ainc mais ne fu si dure mere/Con je par sui. " Elle termine sur une phrase approchante "Fius, je t'envoi ci en escil/C'ainc mere ne fist mais de fil. " qui clôt le couplet d'octosyllabes. De même, son fils la console en lui expliquant qu'elle a agi pour le mieux:

Mere, fait il, laissiés cest plait,

Ne l'avés pas por mon mal fait

Ne por sorfait qui en nous soit. (Éracle, v. 607-609)

Il conclut sur la même idée, "Car je me garirai tres bien ". Les discours sont " parfaits " par cette reprise en écho des mêmes mots à la fin de la réplique. Celle-ci se détache alors en un objet fini, distinct du reste du texte, mis en valeur encore par sa construction rhétorique. Ce phénomène est difficilement compatible avec l'interdépendance des répliques qui accompagne un tempo plus rapide.

Dans le Tristan, la longue taille des répliques a vraisemblablement une source cléricale, elles se caractérisent en effet par leur construction académique. Ainsi, dans cet extrait où deux chevaliers discutent après avoir combattu, l'ordre rigoureux des arguments à l'intérieur de chacune des interventions signale leur aspect scolaire:

Quant [Neronneus] s'est une grant pieche reposés et il a bien s'alainne reprise, il conmenche adont a parler et dist:

"Sire cevaliers, nous nous sommes tant combatu ensamble que vous connissiés auques que je sai faire et a vos armes est bien aparant et a m'espee autresi que je voi tainte et vermeille de vostre sanc. A moi d'autre part apert bien, car je sui plaiés et navrés assés plus que je ne vaurroie. Que vous diroie je? Je ai a cestui point si bien esprouvee vostre forche et vostre bonté conneüe que je di bien en moi meïsmes que vous estes sans doute li mieudres cevaliers et li plus fors que je onques trouvaise de chiaus a qui je me sui esprovés. Et pour ceste cose vauroie je mout volentiers, s'il vous plaisoit, savoir ki vous estes, avant que nous plus feïssom de ceste emprise. "

Mesire Lanselos, quant il a grant pieche escouté le cevalier, il li respont en tel maniere:

21. ROGGER K., "Étude descriptive de la chantefable Aucassin et Nicolette ", Zeitschrift für romanische Philologie, 1951, p. 445. 
«Sire cevaliers, je ai bien entendu que vous avés dit. De ce que vous me faites entendant que a mes armes est aparant li vostre fais et a vostre espee autresi, ki est tainte et vermeille de mon sanc, je vous di bien que de ceste bonté ne vous merchi je pas mout durement. De ceste cose dites vous bien vostre honte et la moie. A vous en donnés los et a moi blasme. Et de ce que vous dites que je sui li mieudres cevaliers que vous onques veïssiés n'ai je pas encore mise l'esprueve avant, car encore n'ai je pas granment moustré que je soie boins cevaliers. Certes, se je fuisse boins cevaliers, je vous eüsse piecha mené dusc'a outranche... " (Tristan 1, 13, 10-37)

La réplique de Neronneus est une demande d'information - il veut connaître le nom de son adversaire - appuyée par une justification: "vous estes sans doute li mieudres cevaliers et li plus fors que je onques trouvaise. " Il appuie cette conclusion sur deux constatations, séparées par « d'autre part»: ses propres armes sont rougies du sang de Lancelot; il est lui-même déjà bien blessé. La réponse de Lancelot constitue comme celle d'un bon opponens, la reprise dans l'ordre de ses arguments. Il réagit aux propos de son adversaire en les citant à chaque fois: "De ce que vous me faites entendant que a mes armes est aparant li vostre fais... je vous di bien que..." et "Et de ce que vous dites que je sui li mieudres cevaliers... " L'ordre rigoureux et la reprise des propos de l'adversaire montre la prégnance du modèle rhétorique scolaire $^{22}$ sur la construction de ces répliques. Par ailleurs, le strict encadrement des répliques par le discours attributif sous forme de verbes placés devant en provient sans doute aussi.

\section{Le nombre de répliques par dialogue}

Le nombre de répliques à l'intérieur d'un dialogue permet d'évaluer son importance relative dans le récit et son degré de mimétisme ${ }^{23}$. Ce nombre a en effet à voir avec la structure plus ou moins complexe du dialogue.

Nous distinguerons pour commencer deux grandes catégories: les dialogues à structure schématique qui ne sont composés que d'un échange - ou de deux échanges dont un est tronqué - et les dialogues à structure complexe qui comprennent au moins deux échanges complets. Quatre ou cinq répliques sont déjà le signe d'une composition sans pour autant prétendre rendre une conversation dans son intégralité. Nous employons ce terme de schématique sans y apporter un jugement

\section{Voir infra p. ???}

23. Là encore on ne prendra pas cette notion de mimétisme de manière systématique: ce n'est pas parce qu'un dialogue est long qu'il est plus mimétique. Nous faisons quotidiennement l'expérience de conversations courtes. Mais un dialogue long peut plus facilement recevoir une structure complexe, proche de la construction non linéaire de la plupart des interactions. 


\begin{tabular}{|c|c|c|c|c|c|c|c|c|}
\hline 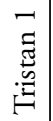 & $\begin{array}{l}\infty \\
\forall^{f}\end{array}$ & $\begin{array}{l}\partial^{\circ} \\
\stackrel{2}{ }\end{array}$ & $\frac{\partial}{\sim}$ & $\stackrel{a}{2}$ & $\begin{array}{l}\partial^{\circ} \\
i n\end{array}$ & $\begin{array}{l}\partial^{\circ} \\
\stackrel{2}{n}\end{array}$ & $\begin{array}{l}\stackrel{0}{ } \\
\infty \\
\sim\end{array}$ & $\begin{array}{l}\partial^{0} \\
i^{2}\end{array}$ \\
\hline 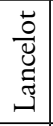 & $\stackrel{\vartheta}{\forall}$ & $\begin{array}{l}\stackrel{0}{\sim} \\
\stackrel{2}{n}\end{array}$ & ஓे & $\begin{array}{l}\stackrel{0}{0} \\
\stackrel{-}{2}\end{array}$ & $\begin{array}{l}a^{0} \\
i n\end{array}$ & $\begin{array}{l}\partial^{0} \\
i n \\
\infty \\
0\end{array}$ & $\begin{array}{l}\text { o̊ } \\
\text { in } \\
\stackrel{\infty}{v}\end{array}$ & 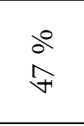 \\
\hline$\stackrel{\Xi}{\sum_{\bar{\Delta}}^{ \pm}}$ & 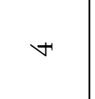 & $\begin{array}{l}\partial^{\circ} \\
\text { ते }\end{array}$ & $\begin{array}{l}\stackrel{\circ}{n} \\
\stackrel{n}{v}\end{array}$ & $\begin{array}{l}\text { a } \\
\text { in }\end{array}$ & $\begin{array}{l}i^{0} \\
i n\end{array}$ & 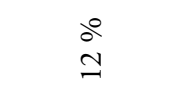 & $\begin{array}{l}\stackrel{0}{\sim} \\
\stackrel{n}{n}\end{array}$ & $\begin{array}{l}\partial^{\circ} \\
n \\
n\end{array}$ \\
\hline 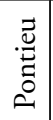 & $\tilde{n}$ & 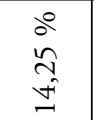 & $\begin{array}{l}\text { o̊ } \\
\text { กิ } \\
\text { nิ }\end{array}$ & 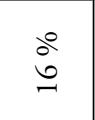 & $\begin{array}{l}\partial^{0} \\
\hat{\sigma}\end{array}$ & 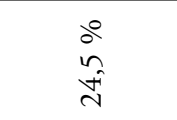 & 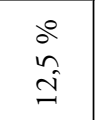 & $\stackrel{\partial^{0}}{n}$ \\
\hline 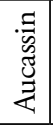 & $\vec{n}$ & $\begin{array}{l}\partial^{\circ} \\
\stackrel{\sim}{\sim}\end{array}$ & $\begin{array}{l}\stackrel{0}{0} \\
\infty \\
-1\end{array}$ & $\begin{array}{l}a^{\circ} \\
a\end{array}$ & $\begin{array}{l}\partial^{\circ} \\
\vec{n}\end{array}$ & $\begin{array}{l}\stackrel{0}{~} \\
\stackrel{\sim}{\sim}\end{array}$ & $\begin{array}{l}\text { वे } \\
\curvearrowleft\end{array}$ & $\begin{array}{l}\stackrel{0}{ } \\
\stackrel{+}{q}\end{array}$ \\
\hline 芉 & $\hat{\mathrm{i}}$ & $\begin{array}{l}\partial^{0} \\
\stackrel{\sim}{\sim}\end{array}$ & $\begin{array}{l}d^{0} \\
\text { ơ }\end{array}$ & 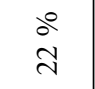 & $\begin{array}{l}\partial^{0} \\
亠^{\prime}\end{array}$ & $\begin{array}{l}\stackrel{0}{0} \\
\hat{0}\end{array}$ & $\begin{array}{l}\partial^{\circ} \\
\text { ñ }\end{array}$ & $\stackrel{0}{0}^{0}$ \\
\hline 㻤 & $\begin{array}{l}0 \\
\stackrel{n}{n}\end{array}$ & $\begin{array}{l}\partial^{0} \\
\tilde{n} \\
\infty\end{array}$ & $\begin{array}{l}\text { o̊ } \\
\text { ñ } \\
\text { - }\end{array}$ & 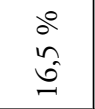 & $\begin{array}{l}\partial^{0} \\
\text { in } \\
\infty \\
n\end{array}$ & 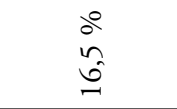 & $\begin{array}{l}\partial^{\circ} \\
\curvearrowleft\end{array}$ & $\begin{array}{l}\partial^{0} \\
\stackrel{n}{F}\end{array}$ \\
\hline 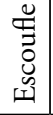 & $\tilde{m}$ & $\begin{array}{l}\partial^{\circ} \\
\vec{F}\end{array}$ & $\begin{array}{l}\text { o̊ } \\
\tilde{n} \\
\tilde{n}\end{array}$ & $\begin{array}{l}\text { o̊ } \\
\text { n̂ } \\
\text { N̂}\end{array}$ & $\begin{array}{l}\stackrel{0}{ } \\
\text { oे }\end{array}$ & $\begin{array}{l}0 \\
\stackrel{2}{n}\end{array}$ & $\begin{array}{l}\alpha^{0} \\
\infty\end{array}$ & $\frac{\partial}{n}$ \\
\hline$\stackrel{\triangleq}{\equiv}$ & $\hat{\imath}$ & $\begin{array}{l}0^{\circ} \\
0 \\
n\end{array}$ & $\begin{array}{l}\stackrel{0}{\hat{n}} \\
\hat{n}\end{array}$ & $\begin{array}{l}\partial^{\circ} \\
\approx \\
\approx\end{array}$ & 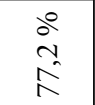 & $\dot{0}_{0}^{\circ}$ & $\begin{array}{l}\sigma^{0} \\
\infty \\
0 \\
0\end{array}$ & 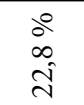 \\
\hline 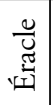 & $\vec{m}$ & $\begin{array}{l}\partial^{\circ} \\
\stackrel{+}{a}\end{array}$ & $\begin{array}{l}\text { o } \\
\tilde{n} \\
\end{array}$ & $\begin{array}{l}\partial^{\circ} \\
i n \\
\infty\end{array}$ & $\begin{array}{l}\partial^{0} \\
\stackrel{N}{N}\end{array}$ & $\begin{array}{l}\partial^{0} \\
=\end{array}$ & $\begin{array}{l}\text { o̊ } \\
\text { in }\end{array}$ & $\begin{array}{l}\text { do } \\
\text { ¿े }\end{array}$ \\
\hline 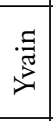 & $\checkmark$ & $\begin{array}{l}\partial^{\circ} \\
n \\
n\end{array}$ & 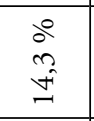 & $\begin{array}{l}a^{\circ} \\
\tilde{n} \\
\tilde{n}\end{array}$ & $\begin{array}{l}\partial^{0} \\
\infty \\
\vec{\sigma}\end{array}$ & $\begin{array}{l}\stackrel{0}{\hat{n}} \\
\hat{n}\end{array}$ & 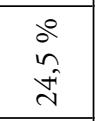 & $\begin{array}{l}\partial^{0} \\
\text { in } \\
\infty \\
n\end{array}$ \\
\hline & 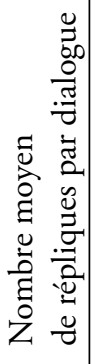 & 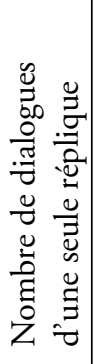 & 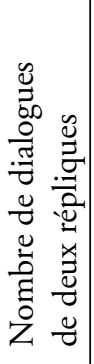 & 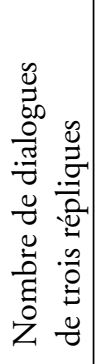 & 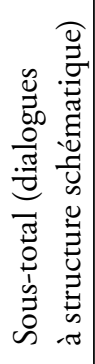 & 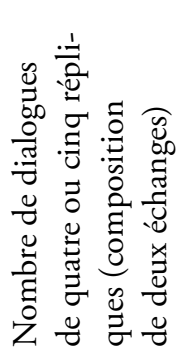 & 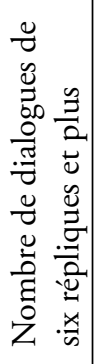 & 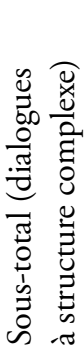 \\
\hline
\end{tabular}


de qualité esthétique. Il indique simplement que le dialogue est peu développé soit parce qu'il est secondaire, ce qui arrive le plus souvent, soit parce qu'il n'est pas dans les habitudes de l'auteur de développer ses dialogue en y ajoutant des répliques. Il pourra leur donner de l'importance par la taille des répliques et leur ornementation rhétorique. Cela n’implique pas une faible implication dramatique. Le dialogue de séparation entre Éracle et sa mère, bien que gardant une structure très simple qui empêche toute péripétie et toute évolution dans les postures, n’en a pas moins une grande résonnance pathétique et didactique.

La construction des interactions dépend des enchaînements des actes de langage à l'intérieur des répliques. Ceux-ci suivent des modèles conventionnels stables, qualifiés de "patterns séquentiels » ${ }^{24}$, qui font reposer l'interaction sur une syntaxe sous-jacente, préconstruite, faisant partie des compétences de tout locuteur :

Il s'agit de successions d'actes de langage ordonnées conventionnellement. Les différentes positions dans la séquence doivent être occupées par des actes de langage d'une certaine catégorie. En général, la progression d'une position à la position suivante correspond à un changement de locuteur. Si un locuteur "fait démarrer " un acte de langage du pattern séquentiel, on attend que son partenaire continue le pattern séquentiel, même si éventuellement il l'interrompt par exemple par une "question au retour » ou par un commentaire qui remet la continuation du pattern à plus tard.

Ces patterns correspondent à des types d'échanges prédéterminés, qui pourront se combiner entre eux dans une certaine mesure ou recevoir des expansions. D. Welke en énumère une vingtaine où le pattern se déroule sur un seul échange à deux mouvements et une dizaine où plusieurs échanges sont combinés. Les dialogues littéraires suivent eux aussi ces patterns, qui donnent une impression de naturel, mais les contraintes narratives contribuent à ajouter d'autres formes aux modèles et tendent à privilégier les structures directives.

La comparaison de trois dialogues appartenant au même motif - la conversation nocturne d'un couple où la dame à force de cajoleries obtient de son mari ou amant la faveur qu'il lui refusait avant - mais construits selon un schéma différent, montrera les conséquences de cette construction sur le développement de la scène.

24. WUNDERLICH D., cité par D. WELKE, «Séquentialité et succès des actes de langage ", DRLAV 22-23, 1980, p. 305. 


\begin{tabular}{|c|c|c|}
\hline Escoufle, (v. 2870-2913) & Vergy, (v. 573-650) & Lancelot, p. 104 \\
\hline $\begin{array}{l}\text { En une couche grant et lee } \\
\text { Se sont andui couchié a aise. } \\
\text { L'empereris l'acole et baise } \\
\text { Et puis les ex et puis la face: } \\
\text { Samblant fait qu'ele velt que face } \\
\text { De li com de sa feme chiere. } \\
\text { He! Dix, cis samblans, ceste } \\
\text { chiere, } \\
\text { N'est pas amors, ains est losenge. } \\
\text { «Sire, fait ele, ja vos ain ge } \\
\text { Plus que mon cors et plus que } \\
\text { m'ame. " } \\
\text { Ahi! Ahi! feme que fame } \\
\text { Com le set ore bien atraire! } \\
\text { Je cuit qu'ele li fera faire } \\
\text { Que ses gens faire ne li porent. } \\
\text { «Sire, fait ele, quant il orent } \\
\text { Geü bras a bras longhement, } \\
\text { Car me dites, por Dieu, comment } \\
\text { Est hui primes venue avant } \\
\text { La parole de vostre enfant } \\
\text { Que Guilliaume aime. Je sai bien, } \\
\text { Certes, ml't desirent son bien } \\
\text { Cil qui heent le mariage, } \\
\text { Por ce qu'il n'est pas de lignage } \\
\text { Qu'il la doie penre n'avoir; } \\
\text { Et neporquant, por nul avoir } \\
\text { Ne remandra qu'il ne soit fait } \\
\text { Dés qu'il vos siet et il vos plaist. } \\
\text { Je sui feme qui n'en puis mais, } \\
\text { Si le m'estuet souffrir em pais; } \\
\text { Mais ml't me grieve toute[s] } \\
\text { voies.» } \\
\text { Tant li moustre essamples et voies } \\
\text { De raison et tant li cort seure } \\
\text { Et en tel point et en tele eure } \\
\text { Que nus ne li peüst veer } \\
\text { Il otroie: es la vos lie }\end{array}$ & $\begin{array}{l}\text { Por ce se tint en itel guise } \\
\text { Que ele mieus le duc atise } \\
\text { A croire que mout soit irie; } \\
\text { Por ce, sanz plus, qu'il l'a besie, } \\
\text { (I) Li dist ele: (a) « Mout estes faus } \\
\text { Et trichierres et desloiaus, } \\
\text { Qui moi moustrez samblant d'amor, } \\
\text { N'oncques ne m'amastes nul jor! } \\
\text { Et j'ai esté lonc tens si fole } \\
\text { Que j'ai creü vostre parole, } \\
\text { Que soventes foiz me disiez } \\
\text { Que de cuer loial m'amiiez! } \\
\text { Més hui m'en sui aperceüe, } \\
\text { Que j'en ai esté deceüe! " } \\
\text { Et li dus dist: (b) "Et vous a quoi? } \\
\text { - (b') Ja me deïstes, par ma foi, } \\
\text { Fet cele qui a mal i bee, } \\
\text { Que je ne fusse si osee } \\
\text { Que je vous enquësse rien } \\
\text { De ce que or savez vous bien. } \\
\text { - (c) De quoi, suer, savez vous, por Dé? } \\
\text { - (c') De ce que cil vous a conté, } \\
\text { Fet ele, mençonge et arvoire, } \\
\text { Qu'il vous a fet pensser et croire. } \\
\text { Més de ce savoir ne me chaut, } \\
\text { Que j'ai penssé que petit vaut } \\
\text { En vous amer de cuer loial: } \\
\text { Que c'onques fust ou bien mal, } \\
\text { Mes cuers riens ne vit ne ne sot } \\
\text { Que ne seüssiez ausi tost; } \\
\text { Et or voi que vous me celez, } \\
\text { Vostre merci, les voz penssez. } \\
\text { Si sachiez ore sanz doutance } \\
\text { Que ja més n'avrai tel fiance } \\
\text { En vous, ne cuer de tel maniere } \\
\text { Com j’ai eü ça en arriere. » } \\
\text { Lors a commecié a plorer } \\
\text { La duchoise et a souspirer, } \\
\text { Et s'esforça plus qu'ele pot. } \\
\text { Et li dus tel pitié en ot }\end{array}$ & $\begin{array}{l}\text { Qant Claudas ot diné, s'asistrent } \\
\text { entre lui et la dame en une } \\
\text { couche, et ele s'encomm[anç]a } \\
\text { a (a) plandre a lui de sa mesaise } \\
\text { et dist: } \\
\text { "Sire, vos i deüssiez bien metre } \\
\text { consoil, car ge n'ai toz cels maus } \\
\text { se por vos non. } \\
\text { - (a') Certes, fait il g'i metroie } \\
\text { mout volentiers consoil (b) se } \\
\text { ge savoie. } \\
\text { - (b') Ge vos enseignerai, fait } \\
\text { ele, coment vos me poez de lui } \\
\text { vengier et vos avocques, (c) se } \\
\text { vos m'amez tant comme j'ai } \\
\text { deservi envers vos. } \\
\text { - (c') De ce, fait il, seiez vos } \\
\text { tote seüre que, se ge puis avoir } \\
\text { lo point, ge vos en vengerai; (d) } \\
\text { et dites comment, se vos saviez, } \\
\text { et ge vos otrois qu'il en iert a } \\
\text { vostre voloir. } \\
\text { - (d') Sire, fait ele, il set de voir } \\
\text { que ce fustes vos qu'il trova en } \\
\text { mon lit gisant, mais senblant } \\
\text { ne noise n'en vost mostrer ne } \\
\text { faire, tant vos redote. Et savez } \\
\text { vos comment vos avez boene } \\
\text { achoison de lui destruire? Il } \\
\text { garde plus a de trois anz les deus } \\
\text { filz au roi Bohort de Gaunes en } \\
\text { une chambre desouz ceste tor, } \\
\text { tant qu'il aient aage et pooir de } \\
\text { vos ocire. Et puis qu'il a ce fait } \\
\text { contre vos, bien a dons a droit } \\
\text { mort deservie. }\end{array}$ \\
\hline
\end{tabular}




\begin{tabular}{|c|c|c|}
\hline Escoufle, (v. 2870-2913) & Vergy, (v. 573-650) & Lancelot, p. 104 \\
\hline $\begin{array}{l}\text { Et dist jamais n'iert aliie } \\
\text { A Guilliaume par mariage. } \\
\text { Di[e]x, quel dolor! Diex, quel } \\
\text { damage! } \\
\text { Com est l'emperere honis! } \\
\text { Or est lie l'empereris } \\
\text { Quant ele ot qu'il n'en avra } \\
\text { mie. } \\
\text { Il s'endort; ele ne dort mie, } \\
\text { Ains l'ataste a sa souef main. }\end{array}$ & $\begin{array}{l}\text { (II) Qu'il li a dit: (d) « Ma bele } \\
\text { suer, } \\
\text { Je ne soufferroie a nul fuer } \\
\text { Ne vostre corouz ne vostre ire; } \\
\text { Més, sachiez, je ne puis pas dire } \\
\text { Ce que volez que je vous die } \\
\text { sanz fere trop grant vilonie. » } \\
\text { Ele respont isnel le pas: } \\
\text { (d') « Sire, si ne m'en dites pas, } \\
\text { Quar je voi bien a cel samblant } \\
\text { Qu'en moi ne vous fiez pas tant } \\
\text { Que celaisse vostre conseil; } \\
\text { Et sachiez que mout me merveil: } \\
\text { Ainc n'oïstes grant ne petit } \\
\text { Conseil que vous m'eüssiez dit, } \\
\text { Dont descouvers fussiez par moi; } \\
\text { Et si vous di, en bone foi, } \\
\text { Ja en ma vie n'avendra. » } \\
\text { Quant ce ot dit, si replora; } \\
\text { Et li dus si l'acole et bese, } \\
\text { Et est de son cuer a malese, } \\
\text { Si que plus ne se pot tenir } \\
\text { De sa volenté descouvrir: } \\
\text { (III) Puis se li a dit: (e) « Bele } \\
\text { dame, } \\
\text { Je ne sai que face, par m’ame, } \\
\text { Que tant m’afi en vous et croi } \\
\text { Que chose celer ne vous doi } \\
\text { Que li miens cuers sache ne ot; } \\
\text { Més, je vous pri, n'en parlez mot: } \\
\text { Sachiez, et itant vous en di, } \\
\text { Que, se je sui par vous trahi, } \\
\text { Vous en receverez la mort. » } \\
\text { Et ele dist (e') « Bien m’i acort! } \\
\text { Estre ne porroit que feïsse } \\
\text { Chose dont vers vous } \\
\text { mespreïsse. » } \\
\text { Cil qui l'aime por ce le croit } \\
\text { Et cuide que veritez soit } \\
\text { De ce que li dist, puis li (f)conte } \\
\text { De sa niece trestout le conte. }\end{array}$ & $\begin{array}{l}\text { - (e) Comment? fait Claudas, } \\
\text { est ce voirs? } \\
\text { - (e') Oil, fait ele, il n'en dotez } \\
\text { pas. Ne vos ne porriez nule } \\
\text { si boene achoison trover sor } \\
\text { lui comme cesti car par tant } \\
\text { a il forfait a estre morz ou } \\
\text { desheritez au mains. } \\
\text { - (a”) Or laissiez atant, fait } \\
\text { Claudas, ne ja ne faites nul } \\
\text { samblant car g'en cuit par } \\
\text { tens mout bien penser et } \\
\text { prochainement. " } \\
\text { Atant prist congié Claudas } \\
\text { et s'an parti de laianz. }\end{array}$ \\
\hline
\end{tabular}


Ces trois dialogues different par leur tempo, leur structure et leur fonction dans le récit. L'extrait du Lancelot est un dialogue à l'importance secondaire qui entraîne finalement assez peu de péripéties, son tempo est rapide $(5,5$ eq. vers/répliques) et il contient un nombre de répliques relativement élevé (huit). L'extrait de l'Escoufle, comme celui de la Châtelaine de Vergy a une grande importance dramatique, puisque la décision ou l'information obtenue vont servir soit d'élément déclencheur soit d'action de résolution dans le schéma narratif de ces deux récits. Cependant, dans l'Escoufle, cette scène reste peu mise en valeur, ce dialogue ne comporte que trois tours de parole dont deux sont rapportés en discours narrativisé et en discours indirect. Cette hiérarchisation des types de discours rend difficile l'établissement d'un tempo significatif. On se bornera à noter qu'il commence très lentement par une longue réplique de 14 vers à laquelle s'ajoutent encore cinq vers de discours narrativisé. Dans la Châtelaine de Vergy, le dialogue, dont on ne présente ici qu'un extrait est largement développé sur onze répliques et a un tempo moyen de 6,5 vers/répliques, mais la taille des répliques diffère beaucoup entre les deux locuteurs. Pour les analyser plus précisément, nous allons étudier l'enchaînement des répliques. Cette première analyse anticipera sur la description détaillée des différentes formes de dialogue.

Construit sur un échange ternaire, le dialogue de l'Escoufle a une structure très simple: [requête (justifiée)/accord/commentaire]. Seule la requête est vraiment développée, mais malgré la mention des efforts que fait l'impératrice pour obtenir cette décision, elle ne reçoit guère d'obstacle. Les efforts sont "dits ", ils sont essentiellement à la charge du narrateur, mais ne sont pas montrés.

Dans le Lancelot, le dialogue suit une structure moyennement complexe qui convient bien pour une petite scène secondaire dans un roman en prose où l'échelle de complexité n'est pas la même: Il a une structure assez classique qui lie, au sein d'un pattern enchâssant [demande de faire/accord] (a-a"), une petite négociation sur les conditions de ce faire $\left\{\mathbf{b b}^{\prime}-\mathbf{c c}\right\}$ - si la dame désire vraiment se venger, c'est à elle de donner à la fois les motifs et les moyens de la vengeance - et une information $\left\{\mathbf{d d}^{\prime}-\mathbf{e e}\right\}$ ' Le dernier échange (ee') n'apporte aucune information supplémentaire, il n'est qu'une confirmation qui vise à donner une impression de naturel et à mettre en scène la surprise de Claudas devant la révélation. Ce dialogue sans être longuement développé, n’en présente pas moins une structure déjà assez complexe visant au plan narratif à mettre en valeur cette révélation : elle est attendue par les échanges de requête qui la précèdent, et prolongée et soutenue par la demande de confirmation qui la suit.

Dans la Châtelaine de Vergy, en revanche, un véritable cycle négociatif se met en place, préparant des retournements de situation à l'intérieur du dialogue. 
Cette négociation cependant n'est pas menée à égalité par les deux locuteurs, la dame la dirige selon une stratégie serrée et habile, intégrant à l'avance dans ses propres répliques les réactions probables de son interlocuteur, comme dans tout dialogue rusé ${ }^{25}$. Elle construit sa stratégie en trois temps: (I) elle commence par un chantage affectif où elle affirme avoir décidé de ne plus aimer son époux. Cette décision n'est pas donnée d'emblée mais construite progressivement à partir des reproches qu'elle lui fait et qui la justifient. En suscitant des demandes de précision par ses informations vagues, elle peut s'approcher de sa décision de manière de plus en plus précise, et en donnant l'impression que ses mots lui sont "arrachés de la bouche " par le duc. Cette première séquence se clôt par une mise en scène physique où les larmes viennent appuyer ses plaintes. (II) Un deuxième temps proprement négociatif intervient alors, portant d'abord sur le bien-fondé du reproche. Le duc réagit à cette décision en réfutant les propos tenus et se défend de l'accusation de cachotterie. La duchesse persistant dans son reproche, la résolution du cycle est négative. (III) Le duc propose alors de lui révéler l'information qu'il connaît, ce qui met fin au conflit. La duchesse lui avait déjà offert cette possibilité par son affirmation répétée de discrétion: s'ouvre alors une dernière partie sur les conditions de mise en œuvre de cette révélation: (e). La révélation (f) peut avoir lieu, qui sera suivie d'une nouvelle demande de serment. Cette fois, le dialogue ne se déroule pas, du moins dans ces deuxième et troisième séquences, selon un pattern traditionnel discernable. Malgré la ruse programmatrice de la duchesse, il semble s'inventer en permanence et se développer par lui-même, ce qui est le signe d'une grande originalité de construction, essentielle pour un dialogue de cette importance.

Ces trois dialogues présentent donc trois degrés de complexité différents en liens avec leur fonction dans le récit - ainsi le dialogue du Lancelot, dont l'importance est secondaire, recherche moins la complexité que celui de la Châtelaine de Vergy, qui constitue l'un des moments forts du texte - et avec les techniques de l'auteur: Jean Renart utilise dans cette scène le dialogue pour illustrer sa narration, et non pour la remplacer. Son dialogue est moins mimétique parce qu’il ne donne pas à voir une situation, mais il la décrit.

Le nombre de répliques dans un dialogue renvoie donc à la complexité plus ou moins grande avec laquelle celui-ci développe la scène. Pour faire passer une même information, pour avoir un effet dramatique à peu près identique, l'auteur peut choisir de faire dialoguer ses personnages très simplement ou peut, au contraire,

25. Sur la ruse, voir DeNOYElle C., "Le discours de la ruse dans les fabliaux », Poétique, n 115 , 1998 , p. 327-350. 
développer son dialogue en y insérant des péripéties, des manipulations, des négociations qui vont contribuer à doter les personnages d'une complexité psychologique et à rendre le passage "naturel » et "vivant ». Sur une structure de base approchante, il peut enrichir son schéma en complexifiant les enchaînements possibles. En reprenant la liste des actes de langage que définit Searle, nous allons décrire maintenant ces différents types d'enchaînement possibles pour montrer quels sont les procédés privilégiés de complexification.

Cette perspective se situe parallèlement à l'étude des types d'échanges ${ }^{26}$ qu'a menée brillamment S. Durrer. Son approche est plus englobante: elle montre le cadre général de construction des dialogues. Les acquis d'une telle étude sont énormes et nous utiliserons ce qu'elle a écrit sur les échanges didactiques, polémiques, dialectiques et phatiques. Nous voulons cependant aller plus loin dans le détail pour voir si l'on peut dégager une " grammaire des conversations " qui rende compte des modèles des narrateurs. En effet, les auteurs médiévaux adoptent des protocoles stéréotypés dans lesquels les enchaînements sont fortement codifiés.

26. S. Durrer n'utilise guère le terme de séquence qui me semble pourtant mieux qualifier la typologie qu'elle a établie. Je préfèrerai donc parler de séquence didactique ou dialectique ou polémique, ou phatique... 